\title{
Linear Control Design, Allocation, and Implementation for the Omnicopter MAV
}

\author{
Yangbo Long and David J. Cappelleri
}

\begin{abstract}
Traditional vertical take-off and landing micro aerial vehicles (VTOL MAVs) are generally underactuated, i.e., equipped with fewer actuators than degrees-of-freedom (DOF). As a consequence, they possess a limited mobility because of the inherent underactuation (e.g., they can neither translate laterally with a zero attitude nor hover at a spot with a nonzero attitude). In this paper, we present the design of a novel MAV, the Omnicopter, with two central counter-rotating coaxial propellers for thrust and yaw control and three perimetermounted variable angle ducted fans to control roll and pitch and provide lateral forces. It can work under two configurations, a fixed $90^{\circ}$ ducted fan angle configuration and a variable angle ducted fan configuration. The variable angle configuration provides full actuation to the Omnicopter position/attitude. After a brief introduction of the Omnicopter platform, we discuss the control design, allocation and implementation for the two configurations separately. Simulations and experimental results verify the performance of the Omnicopter.
\end{abstract}

\section{INTRODUCTION}

Micro aerial vehicles (MAVs) have recently received much interest in the control and robotic communities [1], [2], [3]. This interest is motivated by their potential applications in areas such as search and rescue, surveillance, building exploration, inspection and mapping. Traditional vertical takeoff and landing (VTOL) MAVs are generally underactuated, i.e., equipped with fewer actuators than degrees-of-freedom (DOF), like ducted fan [4], [5], helicopter [6], [7], tricopter [8], [9] and quadrotor [2], [3] configurations.

Historically MAV research has been focused on avoiding interaction with the environment, but several groups are also starting to address the ability for MAVs to manipulate a target or carry objects they encounter [10], [11], [12]. This could greatly expand the types of missions achievable by MAVs. While these results are beneficial, they are implemented on traditional underactuated MAV platforms. In our opinion, an evolution of mechanical design and actuation is necessary in order to realize fully controllable MAVs and achieve complex manipulation tasks.

In this paper we propose a novel actuation concept for a MAV, named the Omnicopter. The Omnicopter design allows for agile movements over the full 6 DOF of the robot. It has two fixed major coaxial counter-rotating propellers in the center used to provide thrust and adjust the yaw angle, and three adjustable angle ducted fans located in three places surrounding the airframe to control its roll and pitch and provide lateral forces. The Omnicopter has two

Y. Long and D. Cappelleri are from the Multi-Scale Robotics and Automation Laboratory, Department of Mechanical Engineering, Stevens Institute of Technology, 1 Castle Point on Hudson, Hoboken, NJ 07030, USA $\{y$ longl, dcappell\}estevens.edu

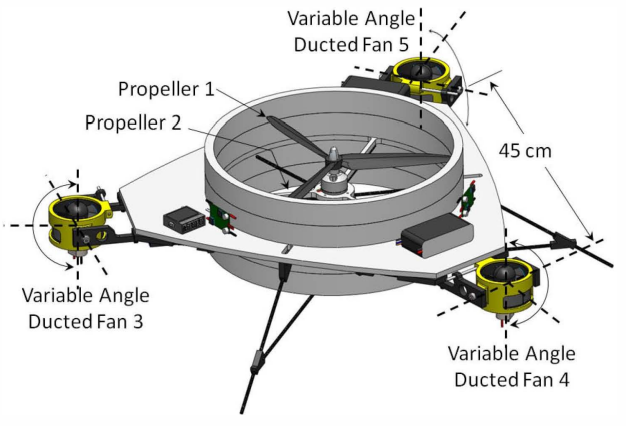

Fig. 1. Omnicopter MAV schematic

configurations: fixed $90^{\circ}$ ducted fan angles and variable ducted fan angles. A schematic of the Omnicopter MAV is shown in Fig. 1.

Similar to some recent work on fully actuated MAVs [13], the Omnicopter MAV has advantages over traditional MAVs. For example, it can maintain zero roll and pitch attitude during lateral translation or arbitrarily orient the fuselage to accomplish complicated grasping tasks. These features are critical in many applications, especially those involving manipulation in cluttered environments where precise position and orientation control is needed.

This paper firstly introduces the building of the Omnicopter platform in Section II, covering the mechanical and electronic setups. Then we present the attitude control, position control and linear control allocation for the fixed $90^{\circ}$ ducted fan angle configuration and the variable angle ducted fan configuration, respectively. Finally, we conclude with simulations and experimental results and a discussion of future perspectives.

\section{OMNICOPTER PLATFORM}

\section{A. Hardware Architecture}

An Omnicopter prototype has been custom-designed and constructed, as shown in Fig. 2 (top). The system consists of the following major components:

- 0.125 " diameter carbon fiber rods along with custom connecting joints laser cut from ABS plastic

- Depron $9 \mathrm{~mm}$ thick hobby foam, laser cut to size

- two 10 x 7 3-blade Master Airscrew propellers

- two BP-U2212/10 BLDC motors and three AEO EDFs

- five Turnigy Plush 25 Amp ESCs

- ArduPilotMega for an IMU and on-board control

- XBee transceiver modules allowing for data logging

- a Thunderpower 3-cell $2700 \mathrm{mAhr}$ Li-Po battery

- a MediaTek MT3329 10Hz GPS for outdoor navigation

- three micro servo motors 


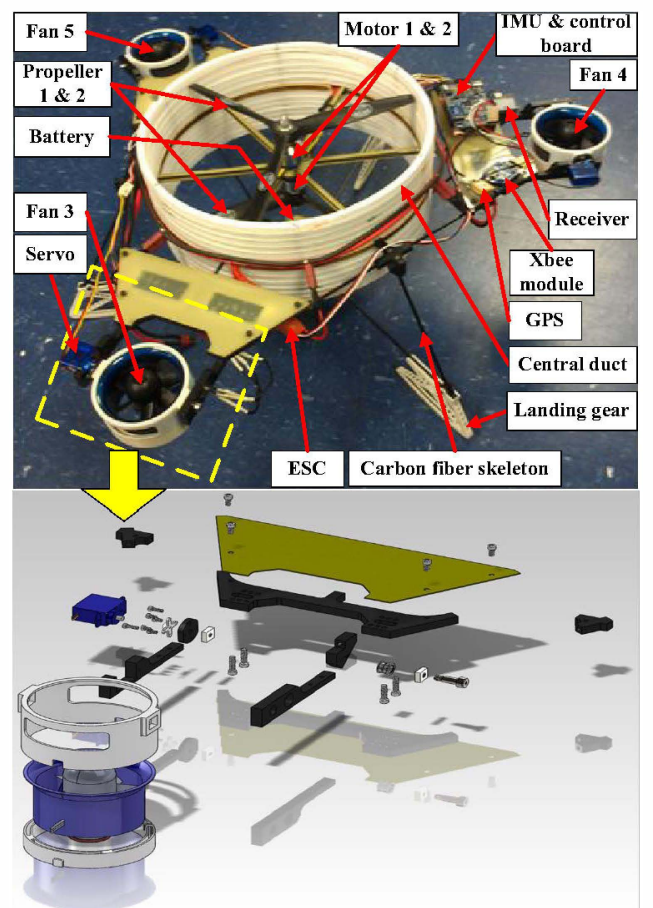

Fig. 2. Omnicopter prototype (top) and thrust vectoring mechanism (bottom)

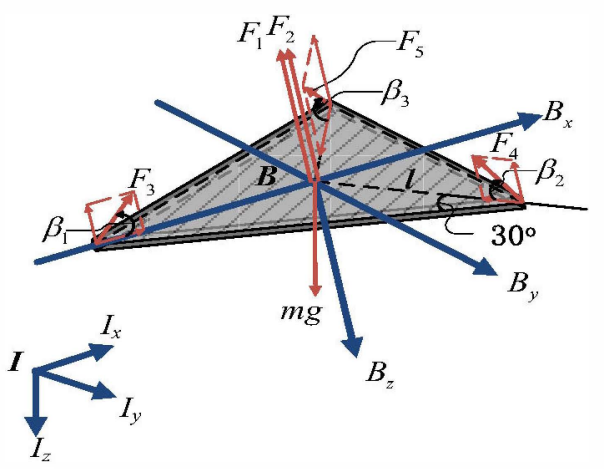

Fig. 3. Free-body diagram

The prototype weighs $2 \mathrm{lbs} 3.5 \mathrm{oz}$. with an available payload at $80 \%$ power of approximately $2 \mathrm{lbs} 6 \mathrm{oz}$. Custom mounts for each of the ducted fans were 3D printed out of ABS plastic. The detailed assembly drawing for the ducted fan thrust vectoring mechanism is shown in Fig. 2 (bottom).

\section{B. Dynamic Model}

Modeling the Omnicopter as a rigid-body, using Newtonian mechanics, let $\boldsymbol{I}=I_{x}, I_{y}, I_{z}$ denote the inertial frame, and $\boldsymbol{B}=\boldsymbol{B}_{x}, \boldsymbol{B}_{y}, \boldsymbol{B}_{z}$ the aircraft body frame, as shown in Fig. 3. Then the model is [14], [15]

$$
\begin{aligned}
& \dot{\boldsymbol{\xi}}=\boldsymbol{v} \\
& m \dot{\boldsymbol{v}}=m_{\boldsymbol{g}} \mathrm{e}_{3}+\boldsymbol{R} f \\
& \dot{\boldsymbol{R}}=\boldsymbol{R} \boldsymbol{\omega}^{\times} \\
& \boldsymbol{J} \dot{\boldsymbol{\omega}}=-\boldsymbol{\omega}^{\times} \boldsymbol{J} \boldsymbol{\omega}+\boldsymbol{\tau}
\end{aligned}
$$

where $\boldsymbol{\xi}, \boldsymbol{v}, m, \boldsymbol{g}, \boldsymbol{R}, \boldsymbol{\omega}, \boldsymbol{J}, f$ and $\boldsymbol{\tau}$ stand for position, velocity, mass, gravitational acceleration, rotation matrix, angular velocity, inertia matrix, and force and torque in the body coordinates, respectively, ${ }^{\times}$denotes the cross product operator for a vector and $e_{3}=\left[\begin{array}{lll}0 & 0 & 1\end{array}\right]^{T}$.

\section{FIXED $90^{\circ}$ DUCTED FAN ANGLE CONFIGURATION}

In the case of the fixed $90^{\circ}$ ducted fan angle configuration, the thrust direction is always along the vertical axis and no lateral force is generated. Therefore, the Omnicopter is essentially underactuated in this configuration.

\section{A. Attitude Control}

We now present two attitude controllers, PD control and integral backstepping control, to follow the desired attitude generated by the position control loop. From (1), the attitude dynamics can be rewritten as

$$
\begin{aligned}
& \dot{\omega}_{x}=a_{1} \omega_{y} \omega_{z}+b_{1} u_{2} \\
& \dot{\omega}_{y}=a_{2} \omega_{x} \omega_{z}+b_{2} u_{3} \\
& \dot{\omega}_{z}=a_{3} \omega_{x} \omega_{y}+b_{3} u_{4}
\end{aligned}
$$

where

$$
\begin{aligned}
& u_{2}=k_{T_{3}}\left(\omega_{5}^{2}-\omega_{4}^{2}\right) \\
& u_{3}=k_{T_{3}}\left[\left(\omega_{4}^{2}+\omega_{5}^{2}\right) \sin 30^{\circ}-\omega_{3}^{2}\right] \\
& u_{4}=k_{Q_{1}} \omega_{1}^{2}-k_{Q_{2}} \omega_{2}^{2}
\end{aligned}
$$

and $a_{1}=\frac{I_{y y}-I_{z z}}{I_{x x}}, a_{2}=\frac{I_{z z}-I_{x x}}{I_{y y}}, a_{3}=\frac{I_{x x}-I_{y y}}{I_{z z}}, b_{1}=\frac{l \cos 30^{\bullet}}{I_{x x}}$, $b_{2}=\frac{l}{I_{y y}}, b_{3}=\frac{1}{I_{z z}}, k_{T_{3}}$ is a thrust factor, $k_{Q_{1}}$ and $k_{Q_{2}}$ are drag factors, and $\Omega=\left[\begin{array}{lllll}\omega_{1} & \omega_{2} & \omega_{3} & \omega_{4} & \omega_{5}\end{array}\right]^{T}$ denotes the five rotors' speed.

1) $P D$ Control: If we apply the small angle approximation, $\phi \approx 0$ and $\theta \approx 0$, the relation between Euler angles' derivatives and body angular speeds can be simplified to be

$$
\left[\begin{array}{c}
\dot{\phi} \\
\dot{\theta} \\
\dot{\psi}
\end{array}\right]=\left[\begin{array}{l}
\omega_{x} \\
\omega_{y} \\
\omega_{z}
\end{array}\right]
$$

Now we linearize (2) about the hovering operating point and write the desired angular accelerations in terms of the new control inputs

$$
\begin{aligned}
& \dot{\omega}_{x}^{\text {des }}=b_{1} u_{2} \\
& \dot{\omega}_{y}^{\text {des }}=b_{2} u_{3} \\
& \dot{\omega}_{z}^{\text {des }}=b_{3} u_{4}
\end{aligned}
$$

where the superscript, des, indicates the desired value.

For the linearized attitude subsystem (4), we design a PD controller to stabilize the attitude of the Omnicopter as below

$$
\begin{aligned}
& u_{2}=k_{\boldsymbol{p}, \boldsymbol{\phi}}\left(\phi^{\text {des }}-\phi\right)+k_{\boldsymbol{d}, \boldsymbol{\phi}}\left(\omega_{x}^{\text {des }}-\omega_{x}\right) \\
& u_{3}=k_{\boldsymbol{p}, \theta}\left(\theta^{\text {des }}-\theta\right)+k_{\boldsymbol{d}, \theta}\left(\omega_{y}^{\text {des }}-\omega_{y}\right) \\
& u_{4}=k_{\boldsymbol{p}, \psi}\left(\psi^{\text {des }}-\psi\right)+k_{\boldsymbol{d}, \psi}\left(\omega_{z}^{\text {des }}-\omega_{z}\right)
\end{aligned}
$$

where control gains $k_{\boldsymbol{p}, \boldsymbol{\phi}}, k_{\boldsymbol{p}, \theta}, k_{\boldsymbol{p}, \psi}$ and $k_{\boldsymbol{d}, \boldsymbol{\phi}}, k_{\boldsymbol{d}, \theta}, k_{\boldsymbol{d}, \psi}$ are chosen to ensure good stabilization performance.

2) Integral Backstepping Control: This section describes the development of an integral backstepping (IB) controller, which is similar to the method described in [16] and [17]. The first step is to consider the tracking error $e_{\boldsymbol{\phi}}=\phi^{\text {des }}-\phi$ and its dynamics

$$
\dot{e}_{\phi}=\dot{\phi}^{d e s}-\omega_{x}
$$


where the angular velocity $\omega_{x}$ is not the control input, $u_{2}$, so we consider it as a virtual control

$$
\omega_{x}^{\text {des }}=k_{1} e_{\phi}+\dot{\phi}^{d e s}+k_{2} \int e_{\phi} d t
$$

This formulation introduces the integral terms into the backstepping design, with $k_{1}$ and $k_{2}$ positive constants. The angular velocity tracking error is defined by

$$
e_{\omega_{x}}=\omega_{x}^{\text {des }}-\omega_{x}
$$

Using (7) we can obtain the derivative

$$
\begin{aligned}
\dot{e}_{\omega_{x}} & =\dot{\omega}_{x}^{d e s}-\ddot{\phi}=k_{1} \dot{e}_{\phi}+\ddot{\phi}^{d e s}+k_{2} e_{\phi}-\ddot{\phi} \\
& =k_{1}\left(\dot{\phi}^{\text {des }}-\omega_{x}\right)+\ddot{\phi} \text { des }+k_{2} e_{\phi}-\ddot{\phi}
\end{aligned}
$$

Using (7) and (8) we can rewrite the roll tracking error dynamics (6)

$$
\begin{aligned}
\dot{e}_{\phi} & =-k_{1} e_{\phi}-k_{2} \int e_{\phi} d t+\omega_{x}^{\text {des }}-\left(\omega_{x}^{\text {des }}-e_{\omega_{x}}\right) \\
& =-k_{1} e_{\phi}-k_{2} \int e_{\phi} d t+e_{\omega_{x}}
\end{aligned}
$$

By replacing $\ddot{\phi}$ in (9) by its linearized attitude dynamics (3) and (4), finally the control input, $u_{2}$, appears in (11)

$$
\dot{e}_{\omega_{x}}=k_{1}\left(\dot{\phi}^{d e s}-\omega_{x}\right)+\ddot{\phi}^{d e s}+k_{2} e_{\phi}-b_{1} u_{2}
$$

The desired dynamics of the angular velocity tracking error is

$$
\dot{e}_{\omega_{x}}=-k_{3} e_{\omega_{x}}-e_{\phi}
$$

which is obtained if the control input is designed to be

$$
\begin{aligned}
u_{2}= & \frac{1}{b_{1}}\left(\left(1-k_{1}^{2}+k_{2}\right) e_{\phi}+\left(k_{1}+k_{3}\right) e_{\omega_{x}}\right. \\
& \left.-k_{1} k_{2} \int e_{\phi} d t+\ddot{\phi}^{d e s}\right)
\end{aligned}
$$

As we can see from the expression of the IB controller, it is essentially a cascaded PID controller. The controllers for pitch and yaw can be derived in a similar manner.

\section{B. Position Control}

Here we present two representative position control methods, hover control and path following control, that use the roll and pitch angles as inputs via a backstepping-like method similar to the approach described in [2]. As we have derived before, the position dynamics are shown as follows

$$
\begin{aligned}
& m \ddot{\xi}_{1}=-(c \psi s \theta c \phi+s \psi s \phi) u_{1} \\
& m \ddot{\xi}_{2}=-(s \psi s \theta c \phi-c \psi s \phi) u_{1} \\
& m \ddot{\xi}_{3}-m g=-c \theta c \phi u_{1}
\end{aligned}
$$

where $u_{1}$ is the thrust along the $-B_{z}$ axis.

1) Hover Control: Here we use pitch and roll angles to control the $(x, y)$ coordinates in the inertial frame. In the hover state, $u_{1} \approx m g$, and under the small angle approximation, we obtain

$$
\begin{aligned}
& \ddot{\xi}_{1}^{\text {des }}=-g\left(\cos \psi^{\text {des }} \theta^{\text {des }}+\sin \psi^{\text {des }} \phi^{\text {des }}\right) \\
& \ddot{\xi}_{2}^{\text {des }}=-g\left(\sin \psi^{\text {des }} \theta^{\text {des }}-\cos \psi^{\text {des }} \phi^{\text {des }}\right) \\
& \ddot{\xi}_{3}^{\text {des }}=g-\frac{1}{m} u_{1}
\end{aligned}
$$

In order to compute the desired attitude for the attitude loop, and the altitude controller $u_{1}$, we calculate the command accelerations, $\ddot{\xi}_{i}$ des , from PID feedback of the position error, $e_{i}=\xi_{i, T}-\xi_{i}$, as

$$
\begin{aligned}
\left(\ddot{\xi}_{i, T}-\ddot{\xi}_{i}^{d e s}\right) & +k_{p, i}\left(\xi_{i, T}-\xi_{i}\right)+k_{i, i} \int\left(\xi_{i, T}-\xi_{i}\right) d t \\
& +k_{d, i}\left(\dot{\xi}_{i, T}-\dot{\xi}_{i}\right)=0
\end{aligned}
$$

where $\xi_{i, T}$ is the desired trajectory we are trying to track, its derivatives $\dot{\xi}_{i, T}=\ddot{\xi}_{i, T}=0$ for hover.

Using (15) and (16), we can compute the desired roll and pitch angles for the attitude controller, from the desired accelerations, as well as the altitude controller $u_{1}$

$$
\begin{aligned}
& \phi^{\text {des }}=-\frac{1}{g}\left(\ddot{\xi}_{1}^{\text {des }} \sin \psi^{\text {des }}-\ddot{\xi}_{2}^{\text {des }} \cos \psi^{\text {des }}\right) \\
& \theta^{\text {des }}=-\frac{1}{g}\left(\ddot{\xi}_{1}^{\text {des }} \cos \psi^{\text {des }}+\ddot{\xi}_{2}^{\text {des }} \sin \psi^{\text {des }}\right) \\
& u_{1}=m g-m \ddot{\xi}_{3}^{d e s}
\end{aligned}
$$

where $\psi^{\text {des }}$ is the predefined desired yaw angle. The desired attitude angles, $\phi^{d e s}, \theta^{d e s}$ and $\psi^{\text {des }}$, are then used as the reference signals for the attitude loop.

2) Path Following Control: Here we use an approach similar to that described in [18] and [19] for path following control. We have a method for calculating the closest point on the trajectory, $\boldsymbol{\xi}_{T}$, to the current position, $\boldsymbol{\xi}$. Let the unit tangent vector of the trajectory associated with that point be $\hat{\boldsymbol{t}}$ and the unit normal vector to the path be $\hat{\boldsymbol{n}}$. We define the position and velocity errors as

$$
\begin{aligned}
& \boldsymbol{e}_{n}=\left(\boldsymbol{\xi}_{T}-\boldsymbol{\xi}\right) \cdot \hat{\boldsymbol{n}} \\
& \dot{\boldsymbol{e}}_{n}=-\dot{\boldsymbol{\xi}} \cdot \hat{\boldsymbol{n}} \\
& \boldsymbol{e}_{t}=\left(\boldsymbol{\xi}_{T}-\boldsymbol{\xi}\right) \cdot \hat{\boldsymbol{t}} \\
& \dot{\boldsymbol{e}}_{t}=\left(\dot{\boldsymbol{\xi}}_{T}-\dot{\boldsymbol{\xi}}\right) \cdot \hat{\boldsymbol{t}}
\end{aligned}
$$

A path following controller is designed by closing the position loop on position errors and velocity errors. We employ PD control in the tangent direction of a path, and PID control in the normal direction of the path.

$$
\begin{aligned}
& \boldsymbol{u}_{t}=\boldsymbol{k}_{p} \boldsymbol{e}_{t}+\boldsymbol{k}_{d} \dot{\boldsymbol{e}}_{t} \\
& \boldsymbol{u}_{n}=\boldsymbol{k}_{p} \boldsymbol{e}_{n}+\boldsymbol{k}_{d} \dot{\boldsymbol{e}}_{n}+\boldsymbol{k}_{i} \int \boldsymbol{e}_{n} d t
\end{aligned}
$$

where $\boldsymbol{u}_{t}=\left[\begin{array}{ll}u_{t, \xi_{1}} & u_{t, \xi_{2}}\end{array}\right]^{T}$ and $\boldsymbol{u}_{n}=\left[\begin{array}{ll}u_{n, \xi_{1}} & u_{n, \xi_{2}}\end{array}\right]^{T}$.

Then the components of the path following control vectors, $\boldsymbol{u}_{t}$ and $\boldsymbol{u}_{n}$, are merged to obtain the desired accelerations

$$
\begin{aligned}
& \ddot{\xi}_{1}^{d e s}=u_{t, \xi_{1}}+u_{n, \xi_{1}} \\
& \ddot{\xi}_{2}^{d e s}=u_{t, \xi_{2}}+u_{n, \xi_{2}}
\end{aligned}
$$

Finally we use (17) to compute the desired roll and pitch angles as well as $u_{1}$.

\section{Control Allocation}

In the case of the fixed $90^{\circ}$ ducted fan angle configuration, five motor speeds need to be computed. The four control inputs, $u_{1}$ to $u_{4}$, are related to the five rotors' speeds, $\omega_{1}$ to 


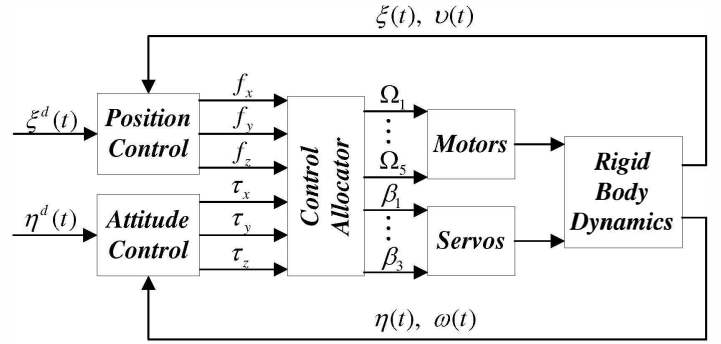

Fig. 4. Control loops for the variable angle ducted fan configuration $\omega_{5}$, by the following equation [14]

$$
\underbrace{\left[\begin{array}{l}
u_{1} \\
u_{2} \\
u_{3} \\
u_{4}
\end{array}\right]}_{\boldsymbol{u}}=\underbrace{\left[\begin{array}{ccccc}
-k_{T_{1}} & -k_{T_{2}} & -k_{T_{3}} & -k_{T_{3}} & -k_{T_{3}} \\
0 & 0 & 0 & -k_{T_{3}} & k_{T_{3}} \\
0 & 0 & -k_{T_{3}} & \frac{k_{T_{3}}}{2} & \frac{k_{T_{3}}}{2} \\
k_{Q_{1}} & -k_{Q_{2}} & 0 & 0 & 0
\end{array}\right]}_{\boldsymbol{A}} \underbrace{\left[\begin{array}{c}
\omega_{1}^{2} \\
\omega_{2}^{2} \\
\omega_{3}^{2} \\
\omega_{4}^{2} \\
\omega_{5}^{2}
\end{array}\right]}_{\boldsymbol{\Omega}}
$$

The above equation can be rewritten in a more compact form as $\boldsymbol{u}=\boldsymbol{A} \boldsymbol{\Omega}$. A natural way to find the desired motors' speeds from the desired command $\boldsymbol{u}$ is the Moore-Penrose inverse matrix method. Since the inverse of $\boldsymbol{A} \boldsymbol{A}^{T}$ exists, the pseudo-inverse of $\boldsymbol{A}$ is given by $\boldsymbol{A}^{\dagger}=\boldsymbol{A}^{T}\left(\boldsymbol{A} \boldsymbol{A}^{T}\right)^{-1}$, and the desired motors' speeds can be calculated according to $\boldsymbol{\Omega}=\boldsymbol{A}^{\dagger} \boldsymbol{u}$.

We wish to determine the desired speeds, $\omega_{i}$, of the five rotors so that $\omega_{\min }^{2} \leq \omega_{i}^{2} \leq \omega_{\max }^{2}, i=1, \cdots, 5$. However, when applying the pseudo-inverse method, we disregard the actuator constraints. There are various ways to accommodate these constraints. The simplest way is to truncate the rotors' speeds by clipping those components that violate some constraints.

\section{VARIABLE ANGLE DUCTED FAN CONFIGURATION}

In the variable angle ducted fan configuration, we use a thrust vectoring mechanism to adjust the orientation of the three ducted fans. We can rewrite and simplify the original dynamic model (1) as

$$
\begin{aligned}
& m \ddot{\xi}_{1}= f_{x} c \theta c \psi+f_{y}(c \psi s \theta s \phi-c \phi s \psi) \\
&+f_{z}(s \phi s \psi+c \phi c \psi s \theta) \\
& m \ddot{\xi}_{2}= f_{x} c \theta s \psi+f_{y}(s \psi s \theta s \phi+c \phi c \psi) \\
&+f_{z}(c \phi s \psi s \theta-s \phi c \psi) \\
& m \ddot{\xi}_{3}= m g-f_{x} s \theta+f_{y} c \theta s \phi+f_{z} c \theta c \phi \\
& \ddot{\phi}=\tau_{x} ; \ddot{\theta}=\tau_{y} ; \ddot{\psi}=\tau_{z}
\end{aligned}
$$

where $f_{x}, f_{y}$ and $f_{z}$ are components of $\boldsymbol{f}, s=\sin$ and $c=$ cos.

\section{A. Control Design}

The control block diagram is shown in Fig. 4. In the virtual control input vector $\boldsymbol{v}=\left[\begin{array}{llllll}f_{x} & f_{y} & f_{z} & \tau_{x} & \tau_{y} & \tau_{z}\end{array}\right]^{T}$, the virtual moment inputs, $v_{4}, v_{5}$ and $v_{6}$, can be designed using standard PD control, to stabilize the attitude subsystem

$$
v_{i}=k_{P_{i}}\left(\eta_{i}^{d}-\eta_{i}\right)+k_{D_{i}}\left(\dot{\eta}_{i}^{d}-\dot{\eta}_{i}\right)
$$

where $i=4,5,6$.
Using (23) and choosing proper gains for the attitude controller, the closed-loop attitude dynamics would converge faster than the closed-loop translational dynamics. When designing a position controller, one can consider the attitude angles, $\psi \approx 0, \theta \approx 0$ and $\phi \approx 0$. Therefore, the translational equations in (22) reduce to

$$
\begin{aligned}
& m \ddot{\xi}_{1}=f_{x} \\
& m \ddot{\xi}_{2}=f_{y} \\
& m \ddot{\xi}_{3}=m g+f_{z}
\end{aligned}
$$

Then, the virtual force inputs, $v_{1}, v_{2}$ and $v_{3}$, can be designed using classical PID control

$$
\begin{gathered}
v_{1}=k_{P_{1}}\left(\xi_{1}^{d}-\xi_{1}\right)+k_{D_{1}}\left(\dot{\xi}_{1}^{d}-\dot{\xi}_{1}\right)+k_{I_{1}} \int_{0}^{t}\left(\xi_{1}^{d}-\xi_{1}\right) d \tau \\
v_{2}=k_{P_{2}}\left(\xi_{2}^{d}-\xi_{2}\right)+k_{D_{2}}\left(\dot{\xi}_{2}^{d}-\dot{\xi}_{2}\right)+k_{I_{2}} \int_{0}^{t}\left(\xi_{2}^{d}-\xi_{2}\right) d \tau \\
v_{3}=k_{P_{3}}\left(\xi_{3}^{d}-\xi_{3}\right)+k_{D_{3}}\left(\dot{\xi}_{3}^{d}-\dot{\xi}_{3}\right) \\
+k_{I_{3}} \int_{0}^{t}\left(\xi_{3}^{d}-\xi_{3}\right) d \tau-m g
\end{gathered}
$$

Therefore, we arrive at the control inputs, $\boldsymbol{v}=$ $\left[\begin{array}{llllll}f_{x} & f_{y} & f_{z} & \tau_{x} & \tau_{y} & \tau_{z}\end{array}\right]^{T}$, in the body coordinate frame.

\section{B. Control Allocation}

In the case of the variable ducted fan configuration, five motor speeds $\left(\omega_{1}\right.$ to $\left.\omega_{5}\right)$ and three servo motor angles $\left(\beta_{1}\right.$ to $\left.\beta_{3}\right)$ need to be computed. The mapping between the

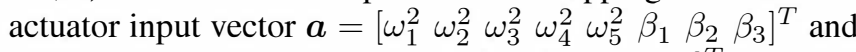
the virtual control input $\boldsymbol{v}=\left[\begin{array}{llllll}f_{x} & f_{y} & f_{z} & \tau_{x} & \tau_{y} & \tau_{z}\end{array}\right]^{T}$ is [14]

$$
\begin{aligned}
& f_{x}=k_{T_{3}}\left(\omega_{3}^{2} c \beta_{1}-\left(\omega_{4}^{2} c \beta_{2}+\omega_{5}^{2} c \beta_{3}\right) s 30^{\circ}\right) \\
& f_{y}=k_{T_{3}}\left(\omega_{5}^{2} c \beta_{3}-\omega_{4}^{2} c \beta_{2}\right) c 30^{\circ} \\
& f_{z}=-k_{T_{1}} \omega_{1}^{2}-k_{T_{2}} \omega_{2}^{2}-k_{T_{3}}\left(\omega_{3}^{2} s \beta_{1}+\omega_{4}^{2} s \beta_{2}+\omega_{5}^{2} s \beta_{3}\right) \\
& \tau_{x}=k_{T_{3}}\left(\omega_{5}^{2} s \beta_{3}-\omega_{4}^{2} s \beta_{2}\right) l c 30^{\circ} \\
& \tau_{y}=k_{T_{3}}\left(\omega_{4}^{2} s \beta_{2}+\omega_{5}^{2} s \beta_{3}\right) l s 30^{\circ}-k_{T_{3}} \omega_{3}^{2} l s \beta_{1} \\
& \tau_{z}=k_{Q_{1}} \omega_{1}^{2}-k_{Q_{2}} \omega_{2}^{2}
\end{aligned}
$$

Solving (26) for $\boldsymbol{a}$, while considering actuator constraints, amounts to performing constrained nonlinear programming. Since control allocation is to be performed in real-time, this may not be computationally feasible. One way to resolve this problem is to linearize (26) locally around $\boldsymbol{a}_{0}$. Then we can arrive at a linear relationship

$$
\boldsymbol{v}(\boldsymbol{a})=\boldsymbol{v}\left(\boldsymbol{a}_{0}\right)+\left.\frac{\partial \boldsymbol{v}}{\partial \boldsymbol{a}}\right|_{a_{\bullet}}\left(\boldsymbol{a}-\boldsymbol{a}_{0}\right)
$$

which leads to the linear control allocation problem

$$
w=B a
$$

where $\boldsymbol{w}=\boldsymbol{v}(\boldsymbol{a})-\boldsymbol{v}\left(\boldsymbol{a}_{0}\right)+\boldsymbol{B} \boldsymbol{a}_{0}$ and the effectiveness matrix $\boldsymbol{B}=\left.\frac{\partial \boldsymbol{v}}{\partial \boldsymbol{a}}\right|_{a_{\bullet}}, \boldsymbol{a}_{0}$ is picked as the previously applied control input, $\boldsymbol{a}(t-\delta)$, with $\delta$ as the step size.

Ducard et al. [20] showed that a control allocation strategy based on the classical approach of a pseudo-inverse matrix only exploits a limited range of the vehicle capabilities to generate thrust and moments. However, a weighted pseudoinverse matrix method is capable of exploiting a much larger domain in the virtual control input $\boldsymbol{w}$. Here we apply the weighted pseudo-inverse method to solve the control 


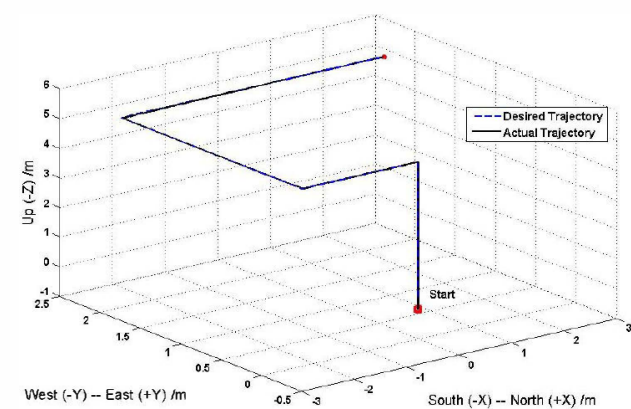

Fig. 5. Path tracking control of the Omnicopter MAV (simulation with fixed $90^{\circ}$ ducted fan angle configuration)
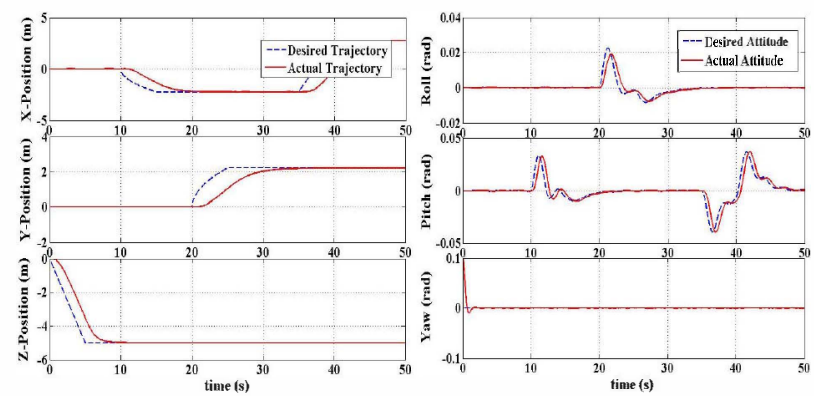

Fig. 6. Path following: position and attitude performance (simulation with fixed $90^{\circ}$ ducted fan angle configuration)

allocation problem. The problem disregarding the actuator constraints is reduced to

$$
\begin{aligned}
& \min _{\boldsymbol{a}}\left\|\boldsymbol{W}_{\boldsymbol{a}} \boldsymbol{a}\right\|_{2} \\
& \text { subject to } \boldsymbol{B} \boldsymbol{a}=\boldsymbol{w}
\end{aligned}
$$

where $W_{a}$ is the weighting matrix.

We can weight each control input with the inverse of its maximum value, $\bar{a}_{i}$, corresponding to $\boldsymbol{W}_{\boldsymbol{a}}=$ $\operatorname{diag}\left(\frac{1}{\bar{a}_{1}}, \cdots, \frac{1}{\bar{a}_{8}}\right)$. After algebraic manipulation we get

$$
a=W_{a}^{-1}\left(B W_{a}^{-1}\right)^{\dagger} w
$$

\section{SIMULATIONS}

Based on the control designs discussed above, simulations are done for the Omnicopter. In the case of the fixed $90^{\circ}$ ducted fan angle configuration, a path following control simulation is shown in Fig. 5. The path consists of a vertical take-off followed by a planar rectangular trajectory. To make the simulations more realistic, random white noise has been added to the position, velocity, attitude and angular velocity measurements. Fig. 6 shows the position and attitude tracking performance. These results prove the effectiveness of the proposed path following controller. (Note: the negative $z$ position means that it's above the ground.)

In order to compare the performance of the Omnicopter under the two configurations, it was tasked to move from its initial position, $\boldsymbol{\xi}=\left[\begin{array}{lll}1 & 1 & 0\end{array}\right]^{T} \mathrm{~m}$, to its final hover position, $\boldsymbol{\xi}=\left[\begin{array}{lll}3 & 3 & 5\end{array}\right]^{T} m$ (see Fig. 7). Its roll and pitch angles are kept at nearly zero in the variable angle ducted fan configuration (Fig. 7 (bottom)), which is impossible for the fixed $90^{\circ}$ ducted fan angle configuration (Fig. 7 (top)). The variable angle ducted fan configuration allows more agility.
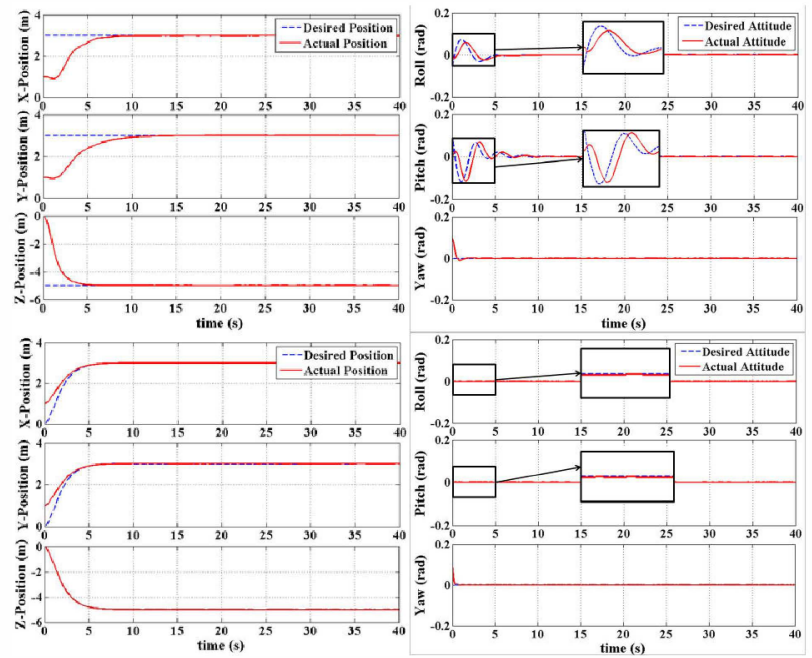

Fig. 7. Hover control: fixed ducted fan angle configuration (top) and variable angle ducted fan configuration (bottom)

\section{EXPERIMENTAL RESULTS}

In this section, we present the real-time experimental results obtained when applying the proposed linear control and allocation techniques to the two Omnicopter configurations described in former sections. The adjustment of the control parameters was carried out following standard methods for tuning a PID control loop.

Fig. 8 - 10 show the experimental results for hovering performance in the fixed $90^{\circ}$ ducted fan configuration and lateral translation performance in the variable angle ducted fan configuration. The top sub-figures show attitude stabilization performance in the fixed ducted fan configuration during hovering, and the bottom ones show lateral translation performance in the variable angle ducted fan configuration for the roll, pitch and yaw angles of the Omnicopter. In the current stage, the attitude control is performed autonomously on-board while the $x, y$ and $z$ positions, as well as the ducted fan angles, are controlled manually using a remote controller. As can be seen from these figures (also see the attached video), the proposed controllers perform well. Because the ducted-fan angles were manually controlled during the experiments, moment unbalance and a decrease in altitude sometimes occur. However, this will be avoided by implementing on-board control allocation, that is a topic of our future work.

\section{CONCLUSIONS}

In this paper, we studied a new MAV configuration called the Omnicopter. Its novel actuation concept allows the three ducted fans to actively rotate about the assembling axes connecting them to the Omnicopter main body, which makes it possible to gain full controllability over its $6 \mathrm{DOF}$.

Because the Omnicopter is underactuated in the fixed $90^{\circ}$ ducted fan angle configuration but overactuated in the variable angle ducted fan configuration, we discussed them separately. Attitude stabilization control, hover/tracking position control and control allocation are designed for the two configurations. A validation of the proposed schemes is provided by means of simulations and experiments. 

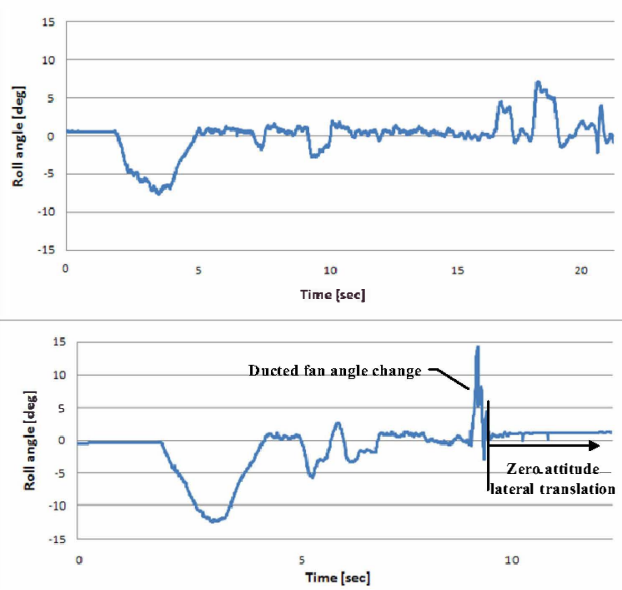

Fig. 8. Roll angle performance: Hovering in the fixed ducted fan configuration (top), and Zero attitude lateral translation in the variable ducted fan configuration (bottom)
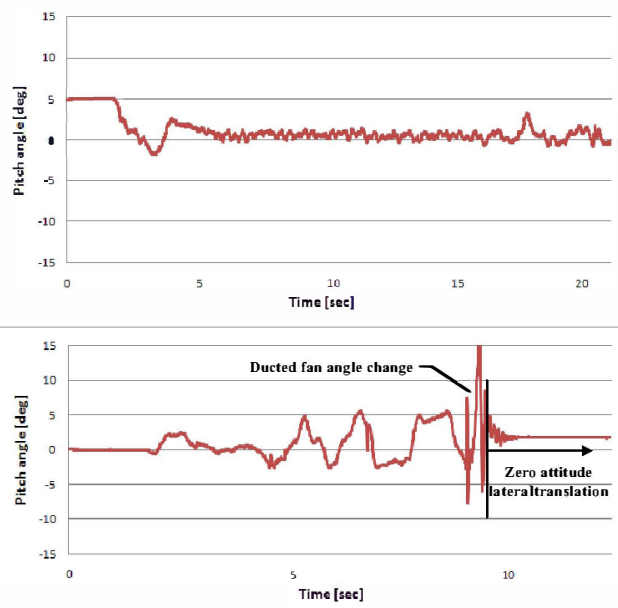

Fig. 9. Pitch angle performance: Hovering in the fixed ducted fan configuration (top), and Zero attitude lateral translation in the variable ducted fan configuration (bottom)
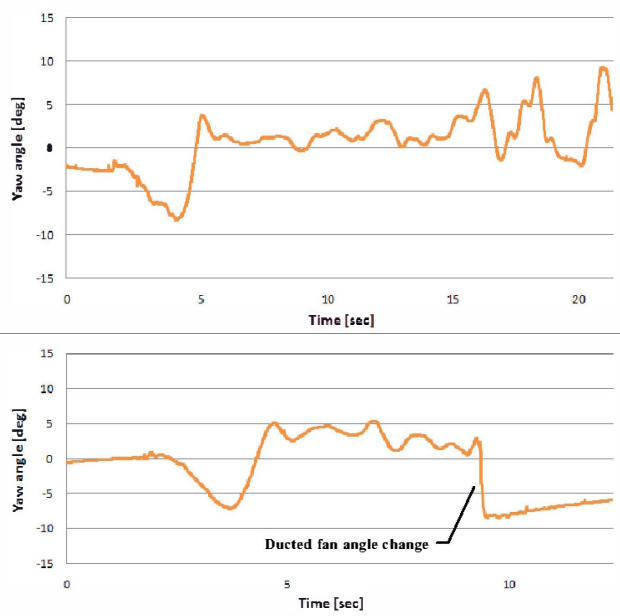

Fig. 10. Yaw angle performance: Hovering in the fixed ducted fan configuration (top), and Zero attitude lateral translation in the variable ducted fan configuration (bottom)

Future work will investigate the implementation of indoor path tracking algorithms by introducing a camera-based localization system, as well as outdoor GPS-guided au- tonomous flights. Additionally, nonlinear optimization techniques can be used as the next step to optimize the control allocation in the variable angle ducted fan configuration.

\section{ACKNOWLEDGMENT}

The authors would like to thank Sean Lyttle, Shi Bai, and Jeremy Wang for the realization and development of the Omnicopter prototype.

\section{REFERENCES}

[1] D. Schafroth, C. Bermes, S. Bouabdallah and R. Siegwart, Modeling, System Identification and Robust Control of a Coaxial Micro Helicopter, in Control Engineering Practice, vol. 18, pp. 700-711, 2010.

[2] N. Michael, D. Mellinger, Q. Lindsey and V. Kumar, The GRASP Multiple Micro UAV Testbed, in IEEE Robotics and Automation Magazine, 2010.

[3] G. Hoffmann, H. Huang, S. Waslander and C. Tomlin, Precision Flight Control for a Multi-vehicle Quadrotor Helicopter Testbed, in Control Engineering Practice, vol. 19, pp. 1023-1036, 2011.

[4] R. Naldi, L. Gentili, L. Marconi and A. Sala, Design and Experimental Validation of a Nonlinear Control Law for a Ducted-fan Miniature Aerial Vehicle, in Control Enginnering Practice, vol. 18, no. 7, pp. 747-760, 2010.

[5] H. Zhao, Development of a Dynamic Model of a Ducted Fan VTOL UAV, Master Thesis, RMIT University, 2009.

[6] B. Ahmed, H. Pota and M. Garratt, Flight Control of a Rotary Wing UAV using Backstepping, in Int. J. Robust Nonlinear Control, vol. 20 , pp. 639-658, 2010.

[7] P. Pounds, D. Bersak and A. Dollar, Stability of Small-scale UAV Helicopters and Quadrotors with Added Payload Mass under PID Control, in Auton Robot, vol. 33, pp. 129-142, 2012.

[8] P. Rongier, E. Lavarec and F. Pierrot, Kinematic and Dynamic Modeling and Control of a 3-Rotor Aircraft, in Proc. IEEE International Conference on Robotics and Automation, pp. 2606-2611, 2005.

[9] J. Escareno, A. Sanchez, O. Garcia and R. Lozano, Triple Tilting Rotor Mini-UAV: Modeling and Embedded Control of the Attitude, in American Control Conference, 2008.

[10] Q. Lindsey, D. Mellinger and V. Kumar, Construction of Cubic Structures with Quadrotor Teams, in Robotics: Science and Systems, 2008.

[11] P. Pounds, D. Bersak and A. Dollar, Grasping from the Air: Hovering Capture and Load Stability, in 2011 IEEE Int. Conf. on Robotics and Automation, pp. 2491-2498, 2011.

[12] M. Orsag, C.Korpela and P. Oh, Modeling and Control of MM-UAV: Mobile Manipulating Unmanned Aerial Vehicle, in J. Intell. Robot Syst., 2012.

[13] M. Ryll, H. Buelthoff and P. Giordano, Modeling and Control of a Quadrotor UAV with Tilting Propellers, in 2012 IEEE Int. Conf. on Robotics and Automation, 2012.

[14] Y. Long, S. Lyttle, N. Pagano and D. Cappelleri, Design and Quaternion-Based Attitude Control of the Omnicopter MAV Using Feedback Linearization, in ASME International Design Engineering Technical Conference (IDETC), 2012.

[15] Y. Long, S. Lyttle and D. Cappelleri, Linear Control Techniques Applied to the Omnicopter MAV in Fixed Vertical Ducted Fan Angle Configuration, in ASME International Design Engineering Technical Conference (IDETC), 2012.

[16] S. Bouabdallah, Design and Control of Quadrotors with Application to Autonomous Flying, PhD Thesis, Ecole Polytechnique Federale De Lausanne, 2007.

[17] F. Hoffmann, N. Goddemeier and T. Bertram, Attitude Estimation and Control of a Quadrocopter, in The 2010 IEEE/RSJ International Conference on Intelligent Robots and Systems, 2010.

[18] G. Hoffmann, S. Waslander and C. Tomlin, Quadrotor Helicopter Trajectory Tracking Control, in AIAA Guidance Navigation and Control Conference and Exhibit, 2008.

[19] V. Ghadiok, J. Goldin and W. Ren, On the Design and Development of Attitude Stabilization, Vision-based Navigation, and Aerial Gripping for a Low-Cost Quadrotor, in Auton Robot, 2012.

[20] G. Ducard and M. Hua, Discussion and Practical Aspects on Control Allocation for a Multi-Rotor Helicopter, in International Archives of the Photogrammetry, Remote Sensing and Spatial Information Sciences, 2011. 